\title{
Câncer de pulmão em trabalhadores expostos à sílica*
}

\author{
Ana Paula Scalia Carneiro ${ }^{1}$, Max Anderson Morales dos Santos ${ }^{2}$, \\ Plínio VASCONCElOS MAIA ${ }^{2}$, SANDHI MARIA BARRETO ${ }^{2}$
}

A sílica é um dos principais agentes ocupacionais relacionados ao câncer de pulmão, sendo classificada pela Agência Internacional para Pesquisa em Câncer (IARC) como substância do grupo 1, ou seja, carcinogênica para humanos, desde 1996. O objetivo deste trabalho é relatar dois casos de trabalhadores previamente expostos à sílica que apresentaram câncer de pulmão, atendidos ambulatorialmente, enfocando pontos relevantes da literatura atual sobre o assunto.

(J Pneumol 2002;28(4):233-236)

\section{Lung cancer in workers exposed to silica}

Silica is one of the main occupational agents associated with lung cancer. It is classified by the International Agency for Research on Cancer (IARC) as a Group 1 substance, i.e., carcinogenic for

humans, since 1996. The objective of this paper is to report two cases of workers previously exposed to silica who developed lung cancer, seen in an out-patient clinic, focusing on important points of the current literature on the subject.

Descritores - Neoplasias pulmonares. Dióxido de Silício. Riscos ocupacionais. Silicose.

Key words - Lung neoplasms. Occupational risk. Silicon dioxide. Silicosis.

\section{INTRODUÇÃO}

No Brasil, e na maioria dos países desenvolvidos, o câncer ocupa a segunda posição como causa de morte em homens, depois da doença coronária, excluindo as causas externas ${ }^{(1)}$. De todos os tipos de câncer, o pulmonar é o mais diagnosticado no mundo, sendo ainda, provavelmente, o mais letal em homens ${ }^{(2)}$. O tabagismo é a causa mais importante do câncer de pulmão, com clara relação dose-resposta e, usualmente, com longo período de latência entre o início e o surgimento do câncer, em

\footnotetext{
* Trabalho realizado no Ambulatório de Doenças Profissionais do Hospital das Clínicas da Faculdade de Medicina da Universidade Federal de Minas Gerais.

1. Doutoranda em Medicina Pública.

2. Aluno de Medicina.

Endereço para correspondência - Dra. Ana Paula Scalia Carneiro, Ambulatório de Doenças Profissionais do Hospital das Clínicas da UFMG, Al. Álvaro Celso, 55, 2º andar - Belo Horizonte, MG . Tel. (31) 32489564; e-mail: apscalia@email.com
}

Recebido para publicação em 31/1/02. Aprovado, após revisão, em 24/4/02.
Siglas e abreviaturas utilizadas neste trabalho

ADP - Ambulatório de Doenças Profissionais

IARC - International Agency for Research on Cancer

OIT - Organização Internacional do Trabalho

OMS - Organização Mundial de Saúde

RX - Radiografia

TC - Tomografia computadorizada

geral em torno de 30 anos. Estima-se que o fumo seja responsável por aproximadamente $85 \%$ dos casos na América do Norte e Europa ${ }^{(3,4)}$ e os demais 15\% sejam devidos a fatores ocupacionais ${ }^{(5)}$; entretanto, os registros de câncer ocupacional no Brasil e em países da América do Sul ainda são muito recentes e escassos(6).

Dos principais agentes ocupacionais implicados na etiologia do câncer de pulmão, a sílica é um dos que envolve maior número de expostos, estimados no Brasil em cerca de seis milhões de trabalhadores ${ }^{(7)}$; entretanto, não se encontrou na literatura nacional nenhuma publicação da associação entre sílica e câncer de pulmão.

O Ambulatório de Doenças Profissionais do Hospital das Clínicas da UFMG (ADP do HC-UFMG) atende, há quase 17 anos, trabalhadores expostos à sílica em diversas atividades profissionais, sendo a maioria proveniente de mineração subterrânea de ouro. Os pacientes comparecem em demanda espontânea ou são encaminhados por outros profissionais, por diversos motivos. Foi realizada uma revisão nos prontuários dos pacientes atendidos no 


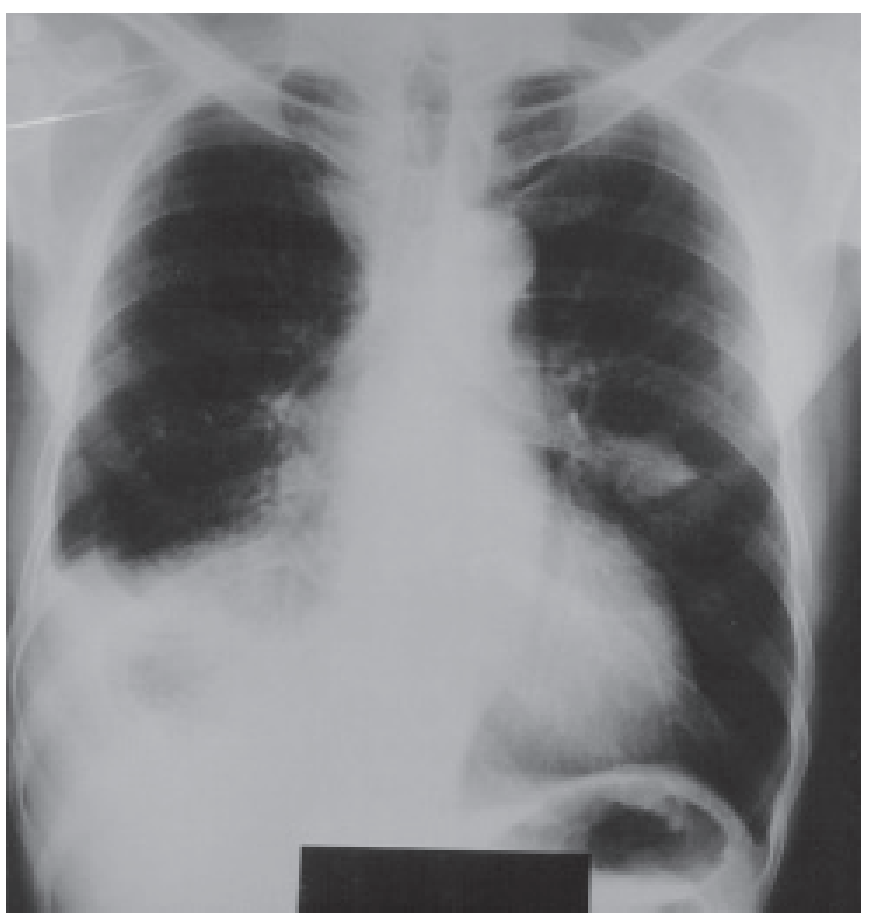

Figura 1 - Caso 1: radiografia de tórax em PA. Lesão nodular perihilar à esquerda, condensação parenquimatosa na base direita e ausência de sinais de silicose.

período de 1989 a 2000 e analisados aqueles que tinham dados completos sobre exposição, radiologia e função pulmonar. Entre os 326 prontuários estudados, identificamos três casos de câncer de pulmão, todos em pacientes do sexo masculino, provenientes de mineração subterrânea de ouro, já afastados da exposição há vários anos. Um deles foi ainda exposto ao amianto e apresentava evidências radiológicas de asbestose; portanto, considerou-se pertinente não inclui-lo neste estudo. Os outros dois casos encontram-se descritos a seguir.

\section{RELATO DOS CASOS}

Caso 1: 67 anos, tabagista desde os 11 anos de idade, minerador durante 30 anos. Compareceu ao ADP para esclarecimento de imagem em RX de tórax. Queixava-se de emagrecimento recente, associado a dores no ombro direito, perna esquerda e região lombar. Negava tosse, expectoração e dispnéia. Ao exame físico apresentava diminuição difusa do murmúrio vesicular. A pesquisa de BAAR foi negativa em várias amostras de escarro. O RX de tórax (Figura 1) mostrou lesão de aspecto nodular na região perihilar esquerda, área de condensação homogênea na base direita com discreto derrame pleural ipsilateral e ausência de sinais de silicose. À TC de tórax (Figura 2) foi vista massa lobulada na língula, com cerca de $5,5 \mathrm{~cm}$ em seu maior diâmetro, espiculada e sem calcificações,

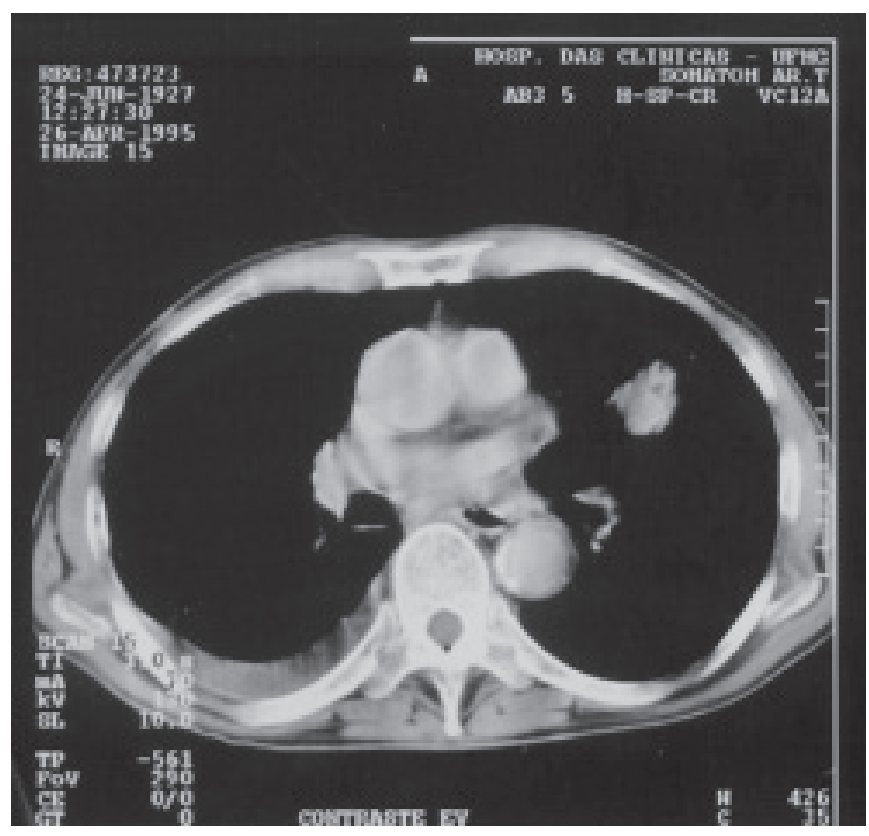

Figura 2 - Caso 1: tomografia computadorizada de tórax. Massa lobulada na língula, espiculada e sem calcificações; derrame pleural à direita.

além de lesões sugestivas de processo inflamatório na base direita, com pequeno derrame pleural homolateral. Não foram vistas lesões sugestivas de silicose. A cintilografia óssea mostrou áreas de hipercaptação na articulação esternoclavicular direita, quinto arco costal esquerdo, vértebras T12 e L5 e articulação sacroilíaca esquerda. Foi realizada fibrobroncoscopia, com um esfregaço positivo para malignidade classe 3 de Papanicolau. Evoluiu com confusão mental, perda de peso e piora clínica. Recebeu alta hospitalar, considerado fora de possibilidade terapêutica, evoluindo para o óbito.

Caso 2: 58 anos, tabagista desde a adolescência, minerador durante 18 anos. Queixava-se de tosse seca, dispnéia aos médios esforços, dor nos joelhos e edema de membros inferiores com 11 meses de evolução. Ao exame encontrava-se eupnéico, com ausculta pulmonar demonstrando apenas diminuição difusa do murmúrio vesicular. O RX de tórax evidenciou micronódulos difusos característicos de silicose. A TC de tórax (Figura 3) evidenciou massa bocelada, com halo de infiltração periférica, sem calcificações, medindo $6,7 \times 5,5 \mathrm{~cm}$, localizada em segmento basal posterior do lobo inferior direito, em contato com o mediastino e coluna vertebral; linfonodomegalia na região subcarinal, além da presença de micronódulos pulmonares difusos, compatíveis com o diagnóstico de silicose. A biópsia pulmonar foi positiva para carcinoma de células escamosas. O paciente foi conduzido para outra instituição, onde evoluiu para o óbito. 


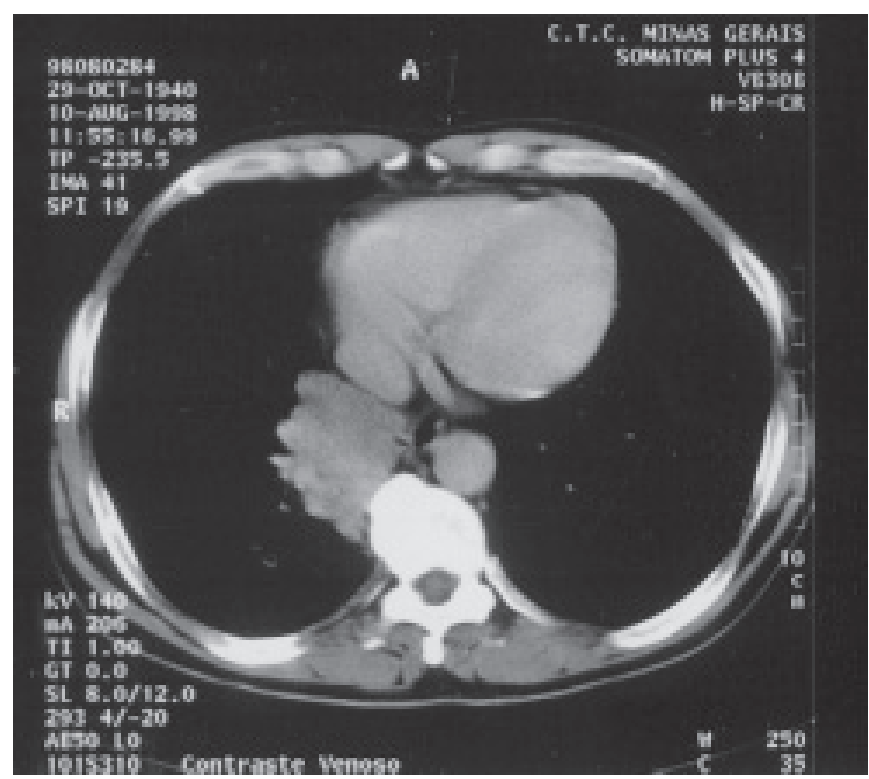

Figura 3 - Caso 2: tomografia computadorizada de tórax. Grande massa bocelada localizada em lobo inferior direito, em contato com coluna vertebral.

\section{DISCUSSÃO}

Nos dois casos descritos os pacientes eram tabagistas de longa data e tinham história de exposição à poeira de sílica por período médio de 30 anos. Apenas um deles apresentou evidência radiológica de silicose (caso 2). $\mathrm{O}$ fato de a confirmação do tipo histológico do câncer de pulmão ter sido feita em apenas um paciente não modifica a possível participação da sílica, já que o tipo histológico $e$ as manifestações clínicas do câncer pulmonar ocupacional em nada diferem dos do câncer não ocupacional. Como se trata de relatos de casos, não se pode afirmar se a sílica foi um fator causal e se agiu de forma isolada ou em combinação com o tabagismo.

A preocupação sobre a sílica e seu possível papel carcinogênico surgiu em 1930, quando um patologista publicou, em Liverpool, uma revisão de 14 casos de silicose em que foram encontrados quatro pacientes com cân$\operatorname{cer}^{(8)}$. Porém, a questão da associação entre exposição à sílica e/ou silicose e câncer de pulmão ainda é polêmica, especialmente devido à coexistência de importantes fatores causais, como o tabagismo e outros carcinogênicos: asbesto, radônio, arsênico e produtos da combustão do diesel, dentre outros. Ainda é discutido se a exposição à sílica per se é suficiente para causar câncer de pulmão embora biologicamente plausível - ou seria necessária a existência da silicose, uma vez que a fibrogênese predispõe à carcinogênese ${ }^{(9)}$. Conforme já citado, apenas um dos casos descritos em nosso estudo apresentava evidências radiológicas de silicose.
A silicose ainda é a principal doença pulmonar ocupacional incapacitante, especialmente em países em desenvolvimento. Nas últimas décadas observou-se modificação da relação entre silicose e/ou exposição à sílica $e$ câncer pulmonar, devido a aumento na expectativa de vida dos trabalhadores expostos à sílica. Esta maior sobrevida deveu-se a dois motivos: a adoção de medidas de controle ambiental, que resultaram em diminuição das formas graves e precoces de silicose, e a terapia antimicobacteriana, que diminuiu a mortalidade por tuberculose pulmonar em pacientes silicóticos ${ }^{(10)}$. Assim, permitiuse observar algumas evidências epidemiológicas que apontaram para risco aumentado de câncer de pulmão em trabalhadores silicóticos ${ }^{(11)}$.

Em outubro de 1996, a IARC passou a classificar a sílica como substância do grupo 1 , concluindo que havia evidências suficientes de carcinogênese em humanos, baseada em estudos concluídos ulteriormente $e^{(12)}$. Dentre eles, um estudo de mortalidade envolvendo 590 trabalhadores expostos à sílica em diversas atividades encontrou risco duas vezes maior de câncer em comparação com a população geral $(\mathrm{SMR}=1,90)^{(13)}$. Outro estudo, de metanálise, mostrou risco relativo para câncer de pulmão de 2,5 entre silicóticos comparados com a população gerall(14). Num estudo de mortalidade com 3.246 trabalhadores expostos à sílica em pedreiras, encontrou-se razão de mortalidade aumentada apenas para os que trabalharam com granito por mais de 20 anos $^{(15)}$.

Recentemente, Martin et al. publicaram um estudo de caso-controle, com 310 casos e 1.225 controles, inseridos em coorte de trabalhadores da indústria de gás e eletricidade da França, encontrando risco 2,3 maior de câncer de pulmão entre os expostos à sílica, com clara relação dose-resposta ${ }^{(16)}$.

Na ausência de tratamento específico efetivo para doenças relacionadas à sílica, a única abordagem é a prevenção, isto é, controle da exposição à sílica. Métodos eficazes de controle incluem supressão da poeira, isolamento no seu processamento e ventilação adequada, substituição por produtos alternativos e, como método temporário e último recurso, o uso de equipamento de proteção individual (EPI). Outra forma de atuar preventivamente poderia ser reforçando campanhas antitabágicas em populações sob riscos ocupacionais, uma vez que podem ocorrer interações entre o fumo e certos agentes ocupacionais ${ }^{(17)}$.

A mortalidade total por câncer de pulmão no Brasil é da ordem de 10.000 homens por ano ${ }^{(18)}$, sendo que a literatura indica risco de duas a três vezes maior nos trabalhadores expostos à sílica, após o ajuste por outros fatores causais como o fumo(13,14,16). Infelizmente, apesar do grande número de expostos à sílica no Brasil, não há dados suficientes para estimar o número de casos de cân- 
cer de pulmão em associação à exposição à sílica, tornando-se imperativo melhorar os registros e informações epidemiológicas, para que possamos dimensionar o pa-

\section{REFERÊNCIAS}

1. Barreto ML. Tendências das doenças crônicas no Brasil. In: Lessa I, editor. O adulto brasileiro e as doenças da modernidade: epidemiologia das doenças crônicas não transmissíveis. Rio de Janeiro: Hucitece, $1998 ; 17$.

2. International Agency for Research on Cancer. Cancer: causes, occurrence and control. Lyon: IARC; 1990. (IARC Scientific Publication, 100).

3. Fraser RS, Muller NL, Colman N, Paré PD. Pulmonary carcinoma. In: Diagnosis of diseases of the chest. $4^{\text {th }}$ ed. Philadelphia: WB Saunders, 1999;1069-228.

4. Ernster VL, Mustacchi P, Osann KE. Epidemiology of lung cancer, In: Murray JF, Nadel JA, editors. Textbook of respiratory medicine. $2^{\text {nd }}$ ed. Philadelphia: WB Saunders, 1994;1504-27.

5. Doll R, Peto R. Causes of Cancer. J Natl Cancer Inst 1981;66:1191308.

6. Algranti E, Menezes AMB, Achutti AC. Lung cancer in Brazil. Semin Oncol 2001;28:143-52.

7. Algranti E. Occupational lung diseases in Brazil. In: Banks DE, Parker $\mathrm{JE}$, editors. Occupational lung diseases: an international perspective. $1^{\text {st }}$ ed. London: Chapman and Hall Medical, 1998;105-15.

8. Dible JH. Silicosis and malignant disease. Lancet 1934;2:986.

9. Weill H, McDonald JC. Exposure to crystalline silica and risk of lung cancer: the epidemiological evidence. Thorax 1996;51:97-102. pel da sílica na etiopatogênese do câncer de pulmão $e$ utilizar os conhecimentos existentes para sua prevenção primária.

10. Balmes J. Silica exposure and tuberculosis: a old problem with some new twiss. J Occup Med 1990;32:114-5.

11. Goldsmith DF, Guidotti TL, Johnston DR. Does occupational exposure to silica cause lung cancer? Am J Ind Med 1982;3:423-7.

12. International Agency for Research on Cancer (IARC). Silica, some silicates, coal dust and para-mid fibrils. Lyon: IARC monograph on the evaluation of the carcinogenic risks to humans; 1997.

13. Goldsmith DF, Beaumont JJ, Morrin LA, Schenker MB. Respiratory cancer and other chronic disease mortality among silicotics in California. Am J Ind Med 1995;28:459-67.

14. Smith AH, Lopipero PA, Barroga VR. Meta-analysis of studies of lung cancer among silicotics. Epidemiology 1995;6:617-24.

15. Costello J, Castellan RM, Swecker GS, Kullman GJ. Mortality of a cohort of U.S. workers employed in crushed stone industry, 19401980. Am J Ind Med 1995;27:625-40.

16. Martin JC, Imbernon E, Goldber M, Chevalier A, Bonenfant S. Occupational risk factors for lung cancer in the French electricity and gas industry. Am J Epidemiol 2000;151:902-12.

17. Algranti E. Tabagismo e ocupação: elo de exposições pouco explorado como estratégia de combate ao tabagismo [editorial]. J Pneumol 2001;27(4):VII.

18. Datasus-MS. Número de mortes por neoplasias de vias respiratórias segundo região. Sexo: masculino. Período: 1998. Disponível em: $<$ http://www.datasus.gov.br/>. Acesso em: 4 jun 2001. 